\title{
Influence of virtual agent politeness behaviors on how users join small conversational groups
}

\author{
Sahba Zojaji \\ zojaji@kth.se \\ EECS, KTH-Royal Institute of \\ Technology \\ Stockholm, Sweden
}

\author{
Christopher Peters \\ chpeters@kth.se \\ EECS, KTH-Royal Institute of \\ Technology \\ Stockholm, Sweden
}

\author{
Catherine Pelachaud \\ catherine.pelachaud@upmc.fr \\ ISIR, Sorbonne University \\ Paris France
}
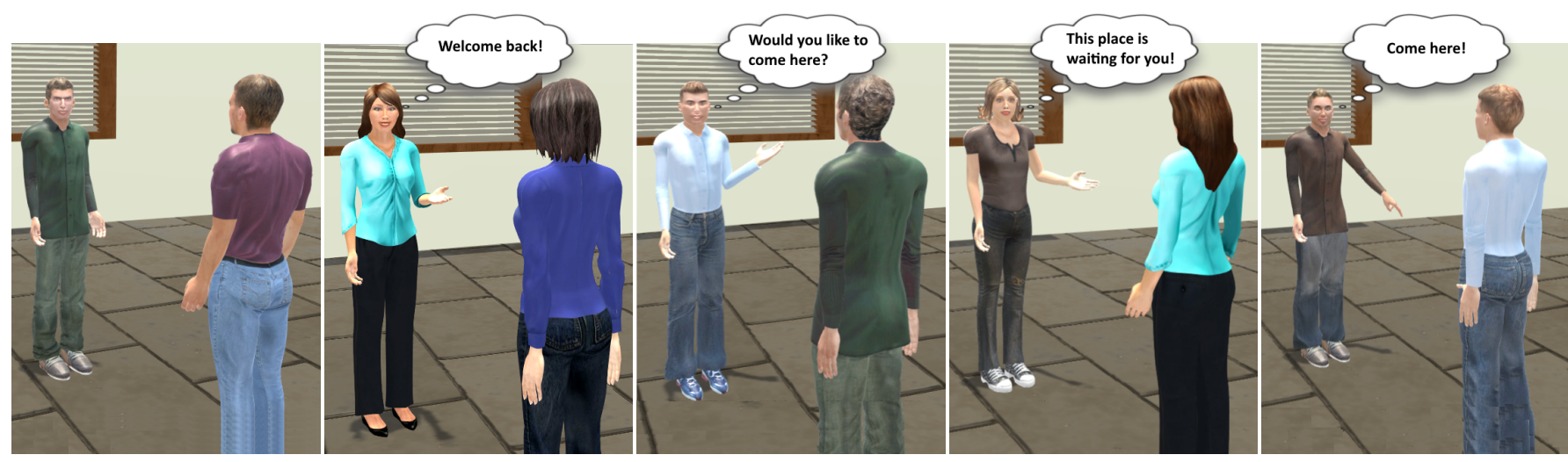

Figure 1: A participant's view of the small group scenario as they approach. The agent shown on the left in each case invites the participant to join the group by conducting verbal and non-verbal behaviors related to the following politeness strategies, from left to right: (a) not face-threatening act (NOT); (b) indirect (IND); (c) negative (NEG); (d) positive (POS); (e) direct (DIR).

\begin{abstract}
Politeness behaviors could affect individuals' decisions heavily in their daily lives and may therefore also play an important role in human-agent interactions. This study considers the impact of politeness behaviors made by a virtual agent, already in a small face-to-face conversational group with another agent, on a human participant as they approach to join it in a virtual environment displayed on a monitor. The agent uses five verbal and nonverbal politeness strategies, ranging from indirect and implicit to direct and explicit, in an attempt to influence the participant to join the group at an inconvenient location, which requires more time and effort than a direct route that would ignore the invitation of the agent. In addition to assessing the success of the strategies at influencing participant behavior, the participants' perception of the agent's persuasive behavior is assessed in relation to clarity, face loss, positive face, and negative face. Based on results from a within-subjects experiment with 30 participants, we found that more direct and explicit politeness strategies have a higher level of success when
\end{abstract}

Permission to make digital or hard copies of all or part of this work for personal or classroom use is granted without fee provided that copies are not made or distributed for profit or commercial advantage and that copies bear this notice and the full citation on the first page. Copyrights for components of this work owned by others than the author(s) must be honored. Abstracting with credit is permitted. To copy otherwise, or republish, to post on servers or to redistribute to lists, requires prior specific permission and/or a fee. Request permissions from permissions@acm.org.

IVA '20, October 19-23, 2020, Virtual Event, Scotland Uk

(c) 2020 Copyright held by the owner/author(s). Publication rights licensed to ACM.

ACM ISBN 978-1-4503-7586-3/20/09 ..\$15.00

https://doi.org/10.1145/3383652.3423917 requesting a participant to join a small group at an inconvenient location, but sometimes negatively impact their perception of the agent. A positive politeness strategy was found to be the most effective for both persuasive success and maintaining a positive impression of the agent.

\section{CCS CONCEPTS}

- Human-centered computing $\rightarrow$ User studies.

\section{KEYWORDS}

Politeness, Face threatening acts, Persuasiveness, Small group behavior, Embodied conversational agents, Multi-modal perception

\section{ACM Reference Format:}

Sahba Zojaji, Christopher Peters, and Catherine Pelachaud. 2020. Influence of virtual agent politeness behaviors on how users join small conversational groups. In IVA '20: Proceedings of the 20th ACM International Conference on Intelligent Virtual Agents (IVA '20), October 19-23, 2020, Virtual Event, Scotland Uk. ACM, New York, NY, USA, 8 pages. https://doi.org/10.1145/ 3383652.3423917

\section{INTRODUCTION}

Politeness plays an important role in humans' social life. It can heavily influence the decision-making of individuals during social interactions. Brown and Levinson [3] defined politeness as an effort to mitigate or avoid doing actions which damage an individual's public self-image, i.e. their face as defined by Goffman [7]. According to their theory, there are five different strategies surrounding 
the use of face-threatening acts to express our needs: not doing a face-threatening act (NOT); off-record or indirect (IND), which uses indirect language to express one's need; negative politeness (NEG) which focuses on avoidance of imposition and giving freedom of action to an addressee; positive politeness (POS) which aims to to avoid giving offense by highlighting friendliness; and bald on-record or direct (DIR) which is a clear, unambiguous and concise way of expressing one's need.

In this study, we investigate the impact of these politeness strategies when used by a virtual agent to invite a participant to join a small conversational group, situated in a virtual environment created using the Unity 3D game engine. In this scenario, two virtual agents are positioned in a face-to-face group configuration (see Figure 1). In all cases, the agent facing the participant invites them into the group as they approach it by looking at them and smiling. In some cases, the agent conducts behaviors associated with politeness strategies to suggest a side or position the participant should move to within the group. In order to understand the effectiveness of the politeness strategies on the persuasive capabilities [2] of the agent, the positions of the agents and participant have been set so that some of the locations suggested by the agent are inconvenient for the participant to reach. They require the participant to take a longer route around a second group member in comparison to walking directly to the closest available position in the group, which is directly in front of them.

We mapped politeness strategies, which range from completely indirect and implicit ways of expressing one's needs, to those that are clear, unambiguous and concise, onto a set of verbal and nonverbal behaviors (see Figure 1 and Table 1) for use by the inviting virtual agent. In Figure 1(a), the agent does not express any further behaviors and thus does not conduct any face-threatening act (NOT). In Figure 1(b), the agent conducts a gesture, moving its left hand by an open palm up as an indirect way (IND) of asking the user to join somewhere on its left side. The open palm sideways of the agent in Figure 1 (c) represents the third politeness strategy (NEG) and is of intermediate clarity with respect to the others. It also gives some freedom to the participant to choose the position at which they join the group. The open palm sideways and partly downward in Figure 1 (d) makes the agent's need clearer while still being friendly, which is in line with the positive politeness strategy (POS). Finally, the most direct nonverbal behavior defined based on the last politeness strategy (DIR) has the agent pointing directly at a specific join position with its index finger.

\section{RELATED WORK}

This study has close links to two domains in the area of human social behavior: group theory and dynamics, and politeness theory. In addition, work has applied these theories to the domain of intelligent virtual agents for the design of small groups of virtual agents that are capable of behaving politely. The following subsections provide related work in each of these areas.

\subsection{Group theory}

When two or more people gather to communicate, they form freestanding conversational groups. Several theories investigate how people manage to share the space around and between them when

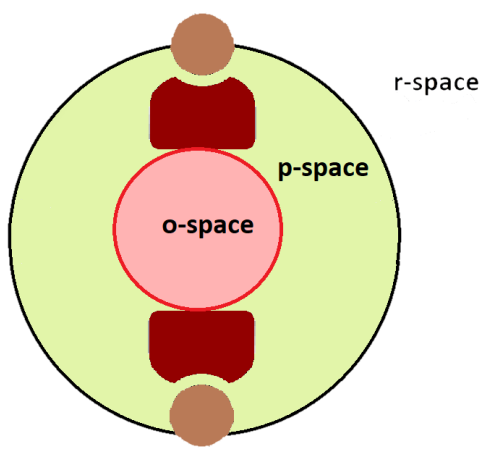

Figure 2: In this study, the participant approaches a small group of two agents in a vis-a-vis F-formation. A newcomer approaches the group from the $r$-space region and is considered to be in the p-space after joining it.

they are a member of a group. Hall [8] described the space surrounding each individual in terms of four distinct zones: (1) intimate space, (2) personal space, (3) social space, and (4) public space. Based on this theory, most of the social interactions between acquaintances occurs in the social space, whereas the personal space is the area for interactions among close friends or family members. This means that people maintain a certain distance to others when they are part of a group. Kendon [11] proposed socio-spatial formations, called F-formations, that describe the common space management in a group in which all members have equal, direct, and exclusive access to the group space. An F-formation is an organisation of three social spaces: o-space, p-space and r-space (Figure 2). The o-space is a convex empty space surrounded by those involved in a social interaction which is exclusive to group members. The p-space is an area surrounding the o-space which contains the group members, while the $\mathrm{r}$-space is the area beyond the $\mathrm{p}$-space for the public.

This study involves a scenario in which two virtual agents are in a vis-a-vis i.e. face-to-face, group formation. A participant-controlled avatar is initialized in the $\mathrm{r}$-space of this group. The participant is asked to approach the group and join it.

\subsection{Small group behaviors in artificial systems}

Multi-agent systems and interactions have received extensive attention in computer and cognitive sciences [18][19]. Within this broader domain, far fewer studies consider those situations in which a newcomer approaches and joins a small group. F-formations and other theories have been applied to computational analysis methods [5] [17] [21], studies [15] and datasets [25] involving small groups. These have formed a basis for the development of artificial models for use by virtual agents and mobile robots that are either members of a group and must make way for newcomers, or are capable of joining a group in a more socially aware manner, for example, by taking into account social body cues, or attempting to minimize intrusions to the group [1] [20] [14] [6] [23] [24].

\subsection{Politeness theory}

According to Brown and Levinson [3], politeness relates to efforts to mitigate or avoid face-threatening acts. A face-threatening act 
(FTA) is a verbal, paraverbal, or non-verbal act which damages an individual's public self-image i.e. their face [7]. Brown and Levinson made a distinction between positive and negative face. Positive face reflects an individual's need for their wishes to be appreciated by others, while negative face reflects an individual's need for freedom of action, freedom from imposition, and the right to make their own decisions. Thus, positive face includes a desire for connection with others, while negative face involves autonomy and independence. There are five main types of politeness strategies that a speaker may choose to enable an addressee to save face:

(1) Not using the face-threatening act (NOT): simply doing no FTAs.

(2) Off-record or indirect (IND): uses indirect language and removes the speaker from the potential to be imposing.

(3) Negative politeness (NEG): avoids imposition on the addressee and is focused on the addressee's negative face.

(4) Positive politeness (POS): attempts to minimize the threat to the addressee's positive face.

(5) Bald on-record or direct (DIR): does not try to minimize the threat to the addressee's face.

A speaker will choose a politeness strategy based on the dangerlevel of a particular FTA. Therefore, the more dangerous FTA will increase the chance of using the lower numbered strategies. The intensity of a face-threatening act is determined by considering the combination of three factors:

- Power: the power difference between the speaker and addressee (superior, subordinate, or at about the same social level);

- Distance: social distance between speaker and addressee (ex. close friend or a distance colleague);

- Rank: ranking of the seriousness of the face threat or sensitivity of a topic within a particular culture.

In the context of politeness in free-standing conversational groups, a newcomer should consider the group and individual spaces if they wish to join on-going conversational group in a polite manner. Typically, a group member may send a signal to a newcomer to invite them to join the group. This invitation could be considered as a face-threatening act and one that could be accomplished in a number of different ways, according to the five politeness strategies described earlier.

In this study, these five politeness strategies were used as a basis for defining five corresponding verbal and nonverbal politeness behaviours used by the virtual agent to invite a newcomer (the participant) to join a group.

\subsection{Politeness and interpersonal attitude in artificial systems}

Social behaviors and norms, such as politeness, and interpersonal attitudes such as friendliness, have been studied in previous research works involving virtual agents. Cafaro et al [4] studied the effect of in-group and out-group friendliness of a group of agents on user's presence and proxemics behavior while joining or passing a group. Linssen et al. [13] investigated how stances corresponds to certain politeness strategies. Lee et al. [12] proposed a high level agent architecture to model and reason polite behavior in social situations involving collision avoidance. However, to the best of our knowledge, there is no work investigating the effect of politeness strategies on the approaching and joining behavior of an individual into a group. More precisely, this work studies the impact of different politeness strategies used by a virtual agent to persuade a newcomer to join the group at a particular side or position, while also accounting for the consequential impact on the perception of the agent's request.

\section{EXPERIMENT}

\subsection{Participants}

Thirty participants with a good proficiency in English were recruited from a population of students and staff at two universities in Sweden and France. The sample included 18 females and 12 males aged between $22-43$ years old $(M=29.33, S D=5.85)$. In terms of participants' knowledge and familiarity with AI systems and IVAs, $20 \%$ declared no knowledge, $27 \%$ basic knowledge, $40 \%$ intermediate knowledge and $13 \%$ advanced knowledge.

\subsection{Scenario}

All participants read a scenario description that was presented on the screen at the beginning of the experiment. The scenario described three adults in a language exchange meeting in an indoor virtual environment. Two of them are intelligent virtual agents in a face-to-face group formation and there is also an avatar representing a human participant. All of them have seen each other before and have already talked together for a while. The avatar left the group for 15 minutes to read more about a topic and is now returning to the group. It should be controlled to join the group.

\subsection{Design}

The experiment used a within-subjects design. The experiment had six conditions which are shown in Table 1. It illustrates all the experiment conditions, their related politeness strategies and explains the verbal and nonverbal behaviors of the agent in each experiment condition. Each participant saw all experiment conditions. The ordering of these conditions were counterbalanced across participants according to a Latin Square design. The six conditions of each experiment were repeated three times in order to increase the robustness of the results. Thus, each experiment consisted of three blocks of the same six conditions in the same order as the first block resulting in a total of 18 trials. The study therefore involved a total 540 trials over all participants and conditions.

The avatar is initialized behind the pre-existing group of two agents that are in a vis-a-vis (face-to-face) group formation (see Figure 3). The group appears rotated with respect to the avatar's starting position. In each trial, the group is rotated either to the left or right so that there is equal variation in the side that the closest and furthest approach points appear. The group and avatar initialization positions and orientations were selected in order to provide a least effort, straightforward path for the participant to join the group. However, the virtual agent which faces the avatar and invites them into the group typically requests them to join the group at the opposite side, which requires substantially more effort to reach. 


\begin{tabular}{llll}
\hline Condition & Strategy & Verbal behavior & Nonverbal behavior \\
\hline \hline 1. Baseline (BSL) & NOT & None & None* \\
2. Indirect (IND) & IND & "Welcome back!" & Open palm up \\
3. Asking (ASK) & NEG & "Would you like to come here?" & Open palm sideways \\
4. Proposing (PRO) & POS & "This place is waiting for you!" & Open palm sideways and partly downward \\
5. Commanding (CMD) & DIR & "Come here!" & Pointing directly at a specific point with the index finger \\
6. Pointing (PNT) & DIR & None & Pointing directly at a specific place with the index finger \\
\hline
\end{tabular}

Table 1: Experiment conditions and their related politeness strategies, verbal and nonverbal behaviors. *Note: In all conditions, the agent conducted the same reaction (gaze at the participant and smile) prior to the politeness behaviours above.

At the beginning of the experiment, participants were briefed that they would participate in a human-agent interaction study and provided consent. They controlled the avatar using a keyboard and viewed the scenario via a monitor. The participant could control the avatar using the up/down keys on the keyboard to move forwards/backwards and they could turn the avatar's body to the right/left via the right/left keys on the keyboard. Therefore, while participants could take a direct route to the closest region $C$ to join the group, their trajectory towards $\mathrm{F}$ was typically piecewise, as exemplified by the orange curve in Figure 3), rather than smooth, as shown by the yellow curve. In each trial, as the participantcontrolled avatar approached the group, a clearly visible agent in the group acknowledged their presence by smiling and looking at them. According to the active condition in the trial, it then engaged in one of the five different verbal and nonverbal behaviors (see Table 1) which were created based on the politeness theory described in Section 2.3. The participant was then free to join the group in any manner they wished. Based on recordings of the movements of participants, Figure 3 shows the most common approximate paths and closest and farthest join regions depending on whether participants moved to the closest $(C)$ or to the farthest $(F)$ region to join the group. The distance that a participant would need to walk in the virtual environment to reach $\mathrm{F}$ is roughly twice that to reach $\mathrm{C}$. Thus, in addition to the constraints of the keyboard control method for moving to $\mathrm{F}$, the path to $\mathrm{F}$ also required greater time and effort to traverse.

After joining the group, the participant was asked to respond to four questions about their perception of politeness of the agent's behavior in that trial. In order to reduce the potential impact of the virtual agents' gender on participants, only female agents were used with female participants, and only male agents were used with male participants. At the end of the experiment, participants were debriefed about the purpose of the study.

\subsection{Stimuli}

An indoor virtual room that was used as a basis for the scenario was developed in Unity 3D game engine ${ }^{1}$. Two Greta ${ }^{2}$ virtual agents were used as the group members in each case, chosen from a set of eight different character appearances, 4 female and 4 male. According to the gender of the participant, two of them were randomly assigned to the group at each trial.

\footnotetext{
${ }^{1}$ http://www.unity3d.com/

${ }^{2}$ https://github.com/isir/greta/wiki
}

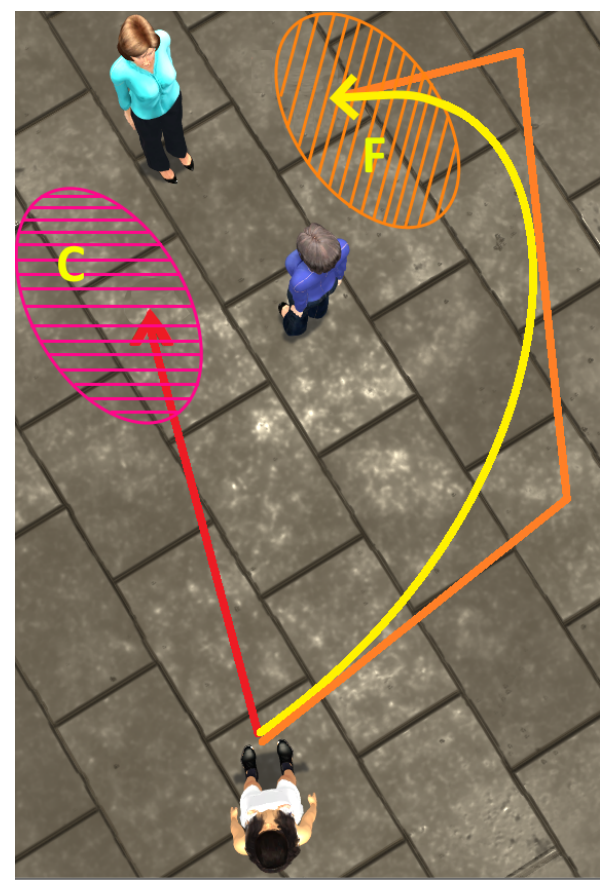

Figure 3: Top-down view of the positioning of the virtual agents (top) and the participant-controlled avatar (bottom). Typical closest $(C)$ and farthest $(F)$ regions for joining the group are shown in addition to a depiction of general approach paths: the direct path to approach $C$ (red line) and a typical path to $F$ (yellow curve). Due to the keyboard control method, the segmented trajectory shown here in orange better represents actual paths to $\mathrm{F}$.

\subsection{Measurements}

An important factor in this study was whether the agent succeeded in persuading the participant to join the group at the farthest region. The boolean value persuasiveness was defined to represent this, with a value $0 / 1$ representing an unsuccessful/successful persuasion attempt in a trial. Another important aspect related to the participants' perception of the politeness of the agent's behavior. This was measured at the end of each trial through a short questionnaire querying the level of agreement of participants with the 
following questions on a 7 point Likert scale, with answers on a scale from "strongly disagree" to "strongly agree":

(1) I could precisely understand the speaker's wants.

(2) I got offended by the speaker's action.

(3) The speaker wanted to increase intimacy with me.

(4) The speaker respected my freedom of action.

Question 1 was designed to evaluate the clarity of agent's request, question 2 was designed to measure loss of face or offensiveness of agent's request, question 3 was designed to verify the level of positive face satisfaction (friendless or warm behavior), and the last question was designed to check the level of negative face satisfaction (respect other's choice, freedom of action, or cold behavior).

\section{RESULTS}

A repeated measures ANOVA with Condition (with six levels, see Table 1) as a within factor and Persuasiveness, Clarity, Offensiveness, Positive politeness and Negative politeness as dependent variables was conducted on the data collected from the study. From the total of 540 trials, 25 trials were discarded since the behaviors of the agent did not activate properly, leaving 515 trials in the final analysis.

Figure 4 illustrates persuasiveness, the success rate (see Table 2) of the agent in each condition at persuading participants to join the group at the farthest region $F$. Of the 515 trials included in the analysis, 425 involved a request to move to the farthest region. The baseline condition ( 90 trials) were not considered to include such a request. Overall, there was a statistically significant effect of Condition on joining the group at the farthest region, $\mathrm{F}(25,2455)=$ $33.850, \mathrm{p}=0.0002$.

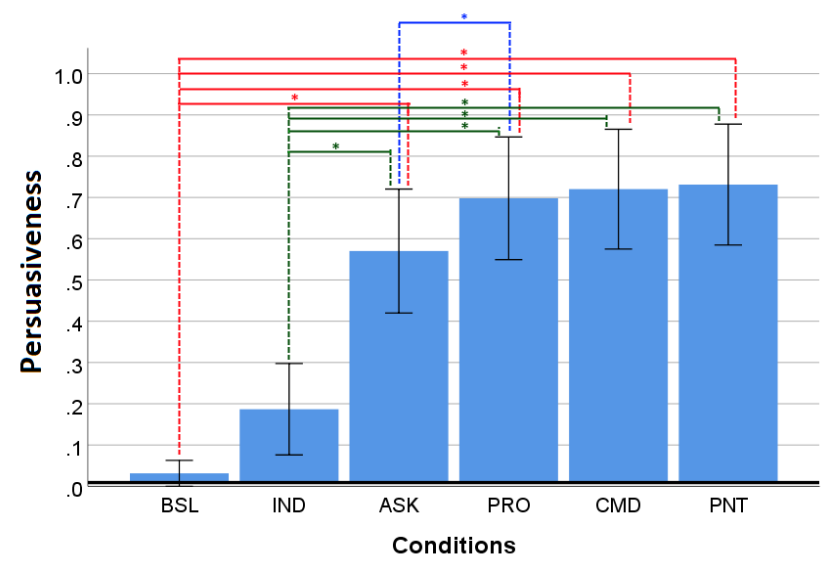

Figure 4: Persuasiveness. Effect of politeness strategy on the participant joining the group at the farthest region $F$. The $Y$ axis plots the average value over all participants and trials of the persuasiveness boolean value. Significance at 0.05 is indicated by *.

A Bonferroni post-hoc comparison suggests that direct strategies are more persuasive, with a significant difference between the conditions BSL, IND and the remainder of the conditions (ASK, PRO, CMD, PNT). Participants were more likely to follow the agent's

\begin{tabular}{llll}
\hline Condition & No. requested & No. successful & Success rate \\
\hline \hline IND & 87 & 14 & $16 \%$ \\
ASK & 83 & 47 & $57 \%$ \\
PRO & 84 & 59 & $70 \%$ \\
CMD & 88 & 64 & $73 \%$ \\
PNT & 83 & 59 & $71 \%$ \\
\hline
\end{tabular}

Table 2: Breakdown of success rate, the number of times per condition that the agent requested and successfully persuaded participants to join the group at the farthest region, $F$. There was a $58 \%$ success rate across all conditions.

recommendation when the agent expressed it by Asking, Proposing, Commanding, or Pointing. In the Baseline (BSL) and Indirect (IND) conditions, participants mostly moved directly to the closest region to join. There was no significant difference between the persuasiveness of agent in the three conditions PRO, CMD and PNT, but there was a significant difference between the ASK and PRO conditions: participants were persuaded significantly more to join to the farthest region when the agent proposed (PRO) for them to join.

Figure 5 shows the effect of politeness strategy on the perception of the clarity of the agent's request. There was a statistically significant effect, with $\mathrm{F}(3.646,105.725)=74.009, \mathrm{p}<.0005$.

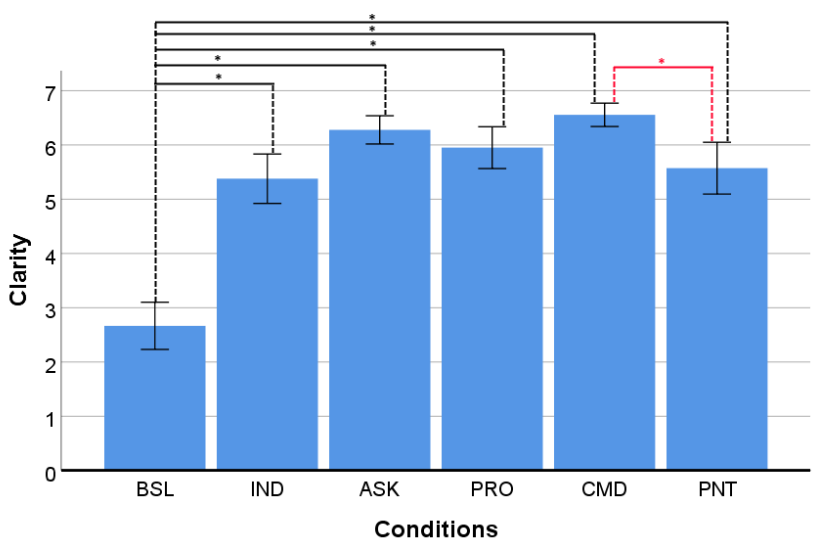

Figure 5: Clarity. Effect of politeness strategy on the perception of the clarity of the request. Significance at 0.05 is indicated by *.

A Bonferroni post-hoc comparison shows that the Baseline (BSL) condition had the least clarity from the participants' perspective: there is a significant difference with all other conditions. Also, there is a significant difference between the Pointing (PNT) and Commanding (CMD) conditions. Surprisingly, commanding (CMD) was rated as having better clarity than Pointing (PNT). There is no significant difference in the clarity of the request between the Asking (ASK) and Proposing (PRO) conditions, or between the Indirect (IND) and Proposing (PRO) conditions.

Figure 6 shows the effect of politeness strategy on the perception of the offensiveness of the agent's request. This relates to the 
participant's loss of face caused by the agent's request. There was a statistically significant effect, with $\mathrm{F}(3.305,95.850)=77.307, \mathrm{p}<$ .0005 .

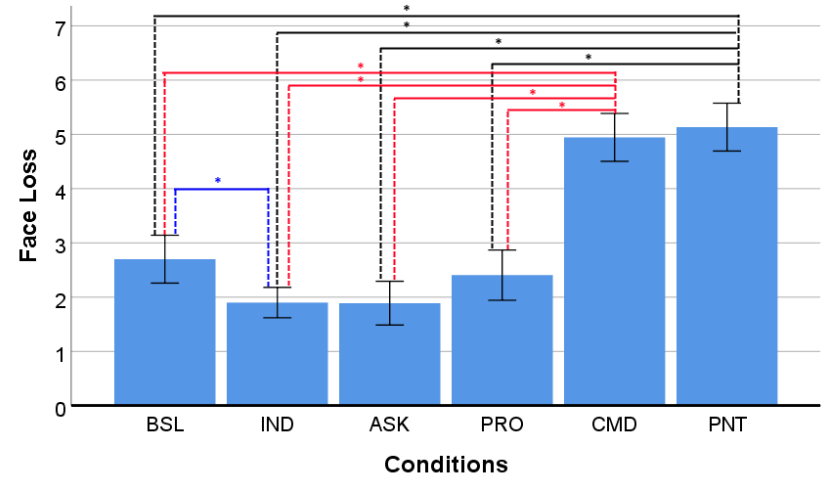

Figure 6: Loss of face. Effect of politeness strategy on the perception of offensiveness of the agent's request. Note: lower values represent less loss of face and are therefore better. Significance at 0.05 is indicated by *.

A Bonferroni post-hoc comparison indicates that the most offensive conditions are the last two conditions: Commanding (CMD) and Pointing (PNT). Also, there is no significant difference between the offensiveness of these two conditions. In addition, participants found the Baseline (BSL) condition more offensive than the Indirect (IND) condition. In the case of BSL, this may be due to a lack of verbal and nonverbal behaviors, since they may have expected the agent to do more than just gaze at them and a smile.

Figure 7 shows the effect of politeness strategy on the perception of the friendliness of the agent's request i.e. positive face. There was a statistically significant effect of the strategy, with $\mathrm{F}(2.910$, $84.396)=26.714, \mathrm{p}<.0005$.

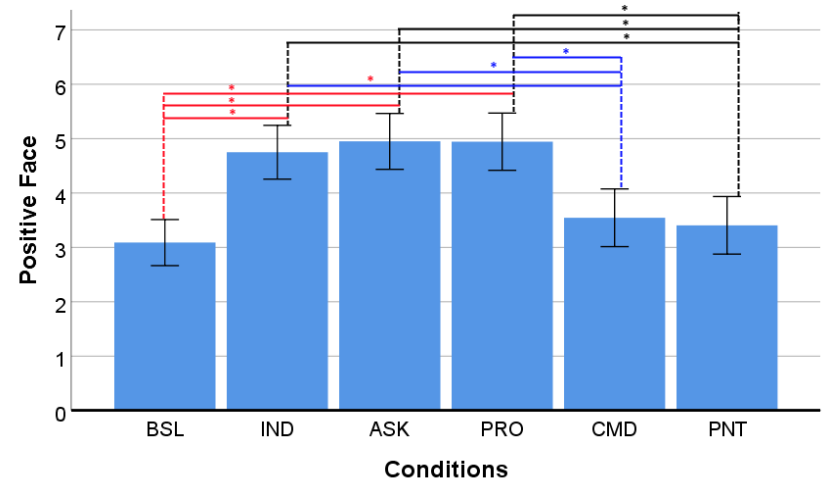

Figure 7: Positive face. Effect of politeness strategy on the perception of the friendliness of the agent's request. Significance at 0.05 is indicated by *.

A Bonferroni post-hoc comparison shows that those conditions perceived to be most friendly by participants and maintain positive face, were Indirect (IND), Asking (ASK), and Proposing (PRO). They have significant differences with the other three conditions. Also, there was no significant difference between the Indirect, Asking, and Proposing conditions in terms of friendliness. In addition, there was no significant difference between the friendliness of the Baseline (BSL), Commanding (CMD), and Pointing (PNT) conditions.

Figure 8 shows the effect of politeness strategy on the perception of freedom of action of the participant. This relates to the impression of how much the agent's request let the participants choose their own position to join the group. There was a statistically significant effect of the strategy on the perception of freedom of action of the participant, $\mathrm{F}(3.077,89.225)=96.935, \mathrm{p}<.0005$.

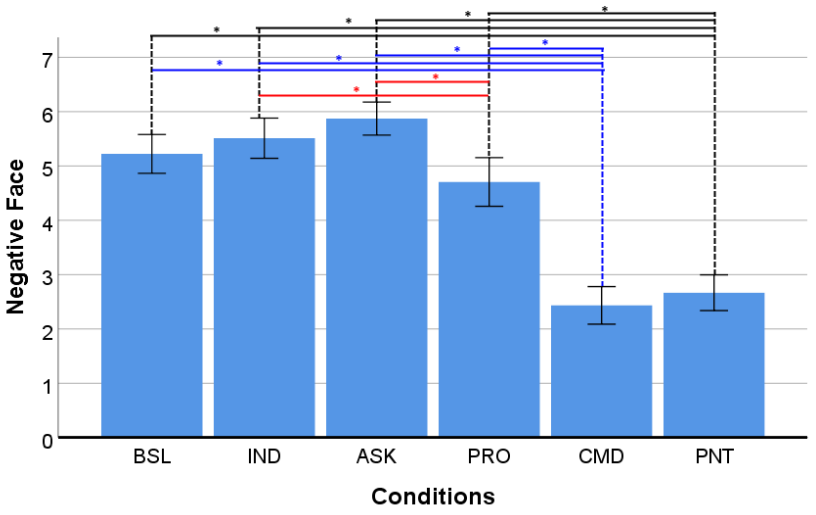

Figure 8: Negative face. Effect of politeness strategy on the perception of freedom of action of the participant. Significance at 0.05 is indicated by *.

A Bonferroni post-hoc comparison shows that the Commanding (CMD) and Pointing (PNT) conditions made participants feel the most constrained in terms of their freedom of action. There is no significant difference between these two conditions on the freedom of action. There was also a significant difference between the Proposing (PRO) condition and the Indirect (IND), Asking (ASK), Commanding (CMD), and Pointing (PNT) conditions. It means that the Proposing condition gave participants less freedom of action than the Indirect and Asking conditions, but more freedom of action than the Commanding and Pointing conditions.

\section{DISCUSSION}

Overall the results suggest that more direct and explicit strategies (ASK, PRO, CMD, PNT) are more persuasive in relation to the participant expending the time and effort required to join the group at an inconvenient location. Research in psychology suggests that, given a choice between similarly rewarding options, humans avoid those that require more work or effort [9]. Generally, this is in line with our findings: When there was no clear instruction or clue about the joining region, participants took the least effort approach and joined the group directly at in the closest region, $C$. A possible reason that more explicit and direct strategies are more persuasive is that they provide clearer clues or goals for participants to follow. While at first glance the reported clarity of the indirect (IND) condition is on par with most of the direct strategies (see Figure 5), it is possible that participants only thought they clearly 
knew what the agent wanted them to do, but they actually did not. This is supported by the persuasiveness scores for the IND condition which are significantly lower than the explicit strategy conditions. The Baseline (BSL) condition was the least successful in terms of persuasiveness and also had the least amount of clarity associated with it. This is not surprising, since the agent only engaged in basic reactive behaviours as the participant approached and did not conduct any further verbal or nonverbal behaviors. Baseline was also perceived as more offensive in relation to loss of face. This might be because the participants expected more behaviors from the agent than eye gaze and a smile. Eye gaze and smile may be important as initial signals to acknowledge the presence of the other, but may not be enough to represent an explicit and clear invitation into the group.

Two of the most persuasive conditions were Commanding (CMD) and Pointing (PNT). However, they were also the most offensive in relation to loss of face and gave the least freedom of action to participants, since they respected the negative face of participants the least. There was also significantly lower clarity in the Pointing than the Commanding conditions. One explanation for this is the lack of any verbal behaviors by the agent in the Pointing condition, which may have reduced certainty about what the agent was requesting in that condition. The specific communication modalities employed by the agent, in isolation or combination, therefore seem to have implications in relation to how they contribute to the directness and clarity of the strategy. The most friendly conditions that respected the positive face of the participants were Indirect (IND), Asking (ASK), and Proposing (PRO). Asking and Proposing conditions still achieved a high success rate for persuasiveness. Especially, Proposing was as successful in terms of persuasiveness as both Commanding (CMD) and Pointing (PNT). Based on these results, Proposing (PRO), which relates to the positive politeness strategy (see Section 2.3), appears to be the best strategy for the virtual agent to persuade participants. It had a relatively high success rate at $70 \%$ (see Table 2) for persuading participants to join the group at the farthest region while avoiding a negative perception of the agent's request. In addition, Proposing did not have a large impact on loss of face (with significant differences to the Commanding and Pointing conditions) and respected the freedom of the participant quite well.

A post-hoc analysis shown in Table 3 provides more details of participant success rates over the course of the experiment. It suggests that participants were fairly consistent in doing what the agent asked them as the experiment progressed. The biggest difference between success rates in blocks 1 and 2 might be explained by a greater familiarisation with the experiment. However, there were no signs that participants were more compliant in the initial block of trials or that their level of compliance wavered in subsequent blocks. Rather, the opposite seemed to be the case. The average success rate of the agent was 58\%. While reasons were provided earlier about why humans may avoid options that require more work or effort, there are also alternative possibilities. One explanation is that since the decision making process requires effort, complying with the agent's request was simply easier in many cases for participants (once they thought they understood it) than to ignore the request. Other psychological studies [10] suggest that effort may also add perceived value so that humans may be more inclined to do such a task.

Participants were also remarkably well-behaved in relation to treating the agents as a social group (see Section 2.1), despite being free to choose any walking path and being informed prior to the study that the virtual agents were autonomous. Participants walked into the p-space of the group to join, but in no cases violated the group's o-space. Nor did any participants take a shortcut of the direct route to the closest region $C$ before moving through the group's o-space and on into the farthest region, $F$ (see Figure 3). This would have satisfied the agent's request while expending less time and effort than the alternative, but would also have been less acceptable in a real social group interaction.

\begin{tabular}{lllll}
\hline Block & Trials & No. requested & No. successful & Success rate \\
\hline \hline 1 & $1-6$ & 137 & 73 & $53 \%$ \\
2 & $7-12$ & 143 & 84 & $59 \%$ \\
3 & $13-18$ & 145 & 88 & $61 \%$ \\
\hline Total & $1-18$ & 425 & 245 & $58 \%$ \\
\hline
\end{tabular}

Table 3: Breakdown of the number of trials per block in which the agent requested and successfully persuaded participants to join the group at the farthest region, $F$.

Overall, an important outcome from the study for this design of persuasive agents relates to choosing strategies that are neither too indirect nor direct when making requests. When indirect methods are used, the nature of the agent's request may not be clearly communicated. On the other hand, while direct methods are clearer, they may be interpreted as being very demanding, making the agents seem unfriendly or socially inept since participants are put in a position that they risk to lose face.

\section{LIMITATIONS AND FUTURE WORK}

This study, like many other studies involving human participants, is dependent on culture. Politeness in terms of a verbal, non-verbal, and/or para-verbal behavior could be expected to be defined differently across cultures. Therefore, participant's perception of politeness in terms of clarity, loss of face, positive face, and negative face, may be quite diverse and questions remain as to how the results in this study might map across cultures. The impact of social context is also likely an important factor. In this study, the agent always acknowledged the participant in some way, which could be interpreted as an invitation into the group. It would be interesting to investigate the impact of different social contexts on participant perception to discover, for example, if participants are similarly offended by direct request strategies from a virtual agent under other circumstances e.g. in an urgent or emergency situation.

In this study, the task that participants were requested to conduct by the agent was purposefully designed to involve more time and effort than such a task might require in a real-world setting. Especially, the input method based solely on the keyboard may not be expected to be representative of natural movement in a real environment. In the future, we are planning to conduct similar studies in Virtual Reality and real environments so that participants can engage in more natural locomotion behavior. 
This relates to our longer term goal of conducting similar experiments with physical robots and small conversational groups in real environments. One challenge with physical robots is that they may have reduced modalities of expression, or the prospect of moving their end effectors may have behavior control, expressivity and/or safety implications. Further studies of the mappings between bodyorienting [22] and gaze motion [16] behaviors and the politeness strategies described here could investigate those behaviors as alternatives or compliments to arm gestures for indicating a side or position to move to.

Additionally, more detailed studies on relationships between the politeness behaviors adopted by group members and the trajectories taken into free-standing conversational groups by newcomers may support existing efforts to develop social aware data-driven navigation algorithms for virtual agents [23] and social robots [24].

\section{CONCLUSION}

The results of this study suggest that more direct and explicit politeness strategies a have higher level of persuasiveness in group scenarios where a newcomer is requested by an agent to join the group at an inconvenient side requiring more time and effort than a direct approach. Our results indicate that more direct strategies, while leading to a higher success rate, may be considered more offensive, less friendly and having less freedom of action by users. Overall, the positive politeness strategy proposing was found to be the most effective for both persuasive success and maintaining a positive impression of the agent with participants. The results from this study provide insights of potential use to the design and modelling of human-agent interaction systems.

\section{ACKNOWLEDGMENTS}

We wish to thank the reviewers for their constructive suggestions that helped to improve the paper. This project has received funding from the European Union's Horizon 2020 research and innovation program under grant agreement No. 765955.

\section{REFERENCES}

[1] P. Althaus, H. Ishiguro, T. Kanda, T. Miyashita, and H. I. Christensen. 2004 Navigation for human-robot interaction tasks. In IEEE International Conference on Robotics and Automation, 2004. Proceedings. ICRA'04. 2004, Vol. 2. IEEE, 18941900

[2] E. André, E. Bevacqua, D. Heylen, R. Niewiadomski, C. Pelachaud, C. Peters, I Poggi, and M. Rehm. 2011. Non-verbal persuasion and communication in an affective agent. In Emotion-oriented systems. Springer, 585-608.

[3] P. Brown and S. C. Levinson. 1978. Universals in language usage: Politeness phenomena. In Questions and politeness: Strategies in social interaction. Cambridge University Press, 56-311.
[4] A. Cafaro, B. Ravenet, M. Ochs, H. H. Vilhjálmsson, and C. Pelachaud. 2016. The effects of interpersonal attitude of a group of agents on user's presence and proxemics behavior. ACM Transactions on Interactive Intelligent Systems (TiiS) 6, 2 (2016), 1-33.

[5] M. Cristani, L. Bazzani, G. Paggetti, A. Fossati, D. Tosato, A. Del Bue, G. Menegaz, and V. Murino. 2011. Social interaction discovery by statistical analysis of Fformations.. In $B M V C$, Vol. 2. 4.

[6] Y. Gao, F. Yang, M. Frisk, D. Hernandez, C. Peters, and G. Castellano. 2018. Social behavior learning with realistic reward shaping. arXiv preprint arXiv:1810.06979 (2018).

[7] E. Goffman. 1967. On face-work. Interaction ritual (1967), 5-45.

[8] E. T. Hall. 1966. The hidden dimension. Vol. 609. Garden City, NY: Doubleday.

[9] C. L. Hull. 1943. Principles of behavior. Vol. 422. Appleton-century-crofts New York.

[10] M. Inzlicht, A. Shenhav, and C. Y. Olivola. 2018. The effort paradox: Effort is both costly and valued. Trends in cognitive sciences 22, 4 (2018), 337-349.

[11] A. Kendon. 1990. Conducting interaction: Patterns of behavior in focused encounters. Vol. 7. CUP Archive.

[12] J. Lee, T. Li, and J. Padget. 2013. Towards polite virtual agents using social reasoning techniques. Computer Animation and Virtual Worlds 24, 3-4 (2013), 335-343.

[13] J. M. Linssen, M. Theune, and D. Heylen. 2013. Taking things at face value: How stance informs politeness of virtual agents. Proc. of CASA (2013).

[14] S. K. Pathi. 2018. Join the Group Formations using Social Cues in Social Robots. In Proceedings of the 17th International Conference on Autonomous Agents and MultiAgent Systems. International Foundation for Autonomous Agents and Multiagent Systems, $1766-1767$.

[15] C. Pedica and H. H. Vilhjálmsson. 2018. Study of Nine People in a Hallway: Some Simulation Challenges. In the 18th Int. Conf. on Intelligent Virtual Agents. ACM, 185-190.

[16] K. Ruhland, C. E. Peters, S. Andrist, J. B. Badler, N. I. Badler, M. Gleicher, B. Mutlu, and R. McDonnell. 2015. A Review of Eye Gaze in Virtual Agents, Social Robotics and HCI: Behaviour Generation, User Interaction and Perception. Comput. Graph. Forum 34, 6 (Sept. 2015), 299-326.

[17] F. Setti, O. Lanz, R. Ferrario, V. Murino, and M. Cristani. 2013. Multi-scale Fformation discovery for group detection. In 2013 IEEE International Conference on Image Processing. IEEE, 3547-3551.

[18] P. Stone and M. Veloso. 2000. Multiagent systems: A survey from a machine learning perspective. Autonomous Robots 8, 3 (2000), 345-383.

[19] R. Sun. 2005. Cognition and multi-agent interaction: From cognitive modeling to social simulation. Cambridge University Press. 1-434 pages.

[20] X. T. Truong and T. D. Ngo. 2018. “To approach humans?": A unified framework for approaching pose prediction and socially aware robot navigation. IEEE Transactions on Cognitive and Developmental Systems 10, 3 (2018), 557-572.

[21] M. Vázquez, A. Steinfeld, and S. E. Hudson. 2015. Parallel detection of conversational groups of free-standing people and tracking of their lower-body orientation. In 2015 IEEE/RSF International Conference on Intelligent Robots and Systems (IROS). IEEE, 3010-3017.

[22] M. Vázquez, E. J. Carter, B. McDorman, J. Forlizzi, A. Steinfeld, and S. E. Hudson. 2017. Towards Robot Autonomy in Group Conversations: Understanding the Effects of Body Orientation and Gaze. In 2017 12th ACM/IEEE International Conference on Human-Robot Interaction (HRI). 42-52.

[23] F. Yang and C. Peters. 2019. App-LSTM: Data-driven Generation of Socially Acceptable Trajectories for Approaching Small Groups of Agents. In Proceedings of the 7th International Conference on Human-Agent Interaction. 144-152.

[24] F. Yang and C. Peters. 2019. AppGAN: Generative Adversarial Networks for Generating Robot Approach Behaviors into Small Groups of People. In 2019 28th IEEE International Conference on Robot and Human Interactive Communication (RO-MAN). IEEE, 1-8.

[25] F. Yang, W. Yin, T. Inamura, M. Björkman, and C. Peters. 2020. Group Behavior Recognition Using Attention-and Graph-Based Neural Networks. In Proceedings of the 24th European Conference on Artificial Intelligence - ECAI 2020. 